\title{
MULTICAO: A SEMANTIC APPROACH TO CONTEXT-AWARE ADAPTATION DECISION TAKING
}

\author{
Vitor Barbosa ${ }^{(1)}$, and Maria Teresa Andrade $(1),(2)$ \\ (1) INESC Porto, (2) FEUP, Rua Dr. Roberto Frias 378, 4200-465 Porto, Portugal \\ Email: \{vhsb, maria.andrade\}@inescporto.pt
}

\begin{abstract}
The use of context is already a reality in different application fields. One such example is the Robotics field, where sensors are used to capture information about the surrounding environment, allowing the system to react to changes. But also in the multimedia communications field, some work has recently emerged to allow combining sensed context and use that combined information to decide how to adapt the content. This paper describes the MULTICAO ontology, which lies at the core of an Adaptation Decision Engine, providing it with a formal description of the surrounding environment in a similar way as humans would describe it. This way the system has higher chances to take adaptation decisions that best meet users' expectations.
\end{abstract}

\section{INTRODUCTION}

Nowadays, it is no longer so unusual to find applications, systems or devices that are aware of the context. Sensors are used to collect information about the characteristics or conditions of the surrounding environment. However, those systems tend to use directly the acquired context, reacting to sensed changes in an individual manner. Some approaches are exploring the use of ontologies to enable a formal description of high-level notions of contexts, in a similar way as humans describe real-world situations. This paper describes one such approach, applicable to multimedia networked applications, where a two-layer ontology enables the formal characterization of generic context as well as the extension to specific application scenarios. Low-level context is acquired and then used to infer a high-level notion of context using rules appropriate to specific application scenarios. The system is thus able to recognize with more detail the situation in which the consumption of multimedia content is occurring. This ontology, designated as MULTICAO, is at the core of an Adaptation Decision Engine (ADE), which decides the type of adaptation to be performed on the multimedia content, selecting appropriate encoding parameters, including frame resolution and rate, encoding format, target bit rate, audio volume etc. This ADE is being developed within the framework of the European project VISNET-II as part of a context-aware content adaptation platform for Virtual Collaboration.
Approaches for content adaptation decision have already been proposed by some research groups [1], [2] . These frameworks are built around the MPEG-21 standard [7], whereby the decision taking operation is focused on solving a constraints matching problem. The constraints, imposed by the conditions of the context of usage, are treated individually and no relations are established between them, or with the characteristics of the application or service. Although our approach still builds on MPEG-21, we have incorporated the use of ontologies to create and share formal knowledge from acquired context. More importantly, to allow to create additional knowledge through reasoning using rules and relationships specified in the ontology.

This paper is organised as follows. Section 2 briefly describes ontology and metadata tools relevant to this work. Section 3 describes the ADE architecture, whereas section 4 provides details on the MULTICAO ontology. Section 5 explains how the ontology can be used in a specific application scenario. Finally, section 6 draws the conclusions.

\section{ONTOLOGIES IN CONTEXT-AWARE CONTENT ADAPTATION}

Most of current context-aware applications use contextual information directly acquired from sensors to react to changes. However, combinations of the same information may lead to different conclusions and may thus be interpreted as characterizing different real-world situations which requiring alternative reactions. Systems that can actually build this kind of additional knowledge will be in a better position to suggest and implement more appropriate adaptations [3]. The new generation of context-aware systems is using ontologies to build this additional knowledge [4], [5]. An ontology-based approach to model context provides the means to formalise concepts from acquired context and to specify relations and rules between those concepts. Accordingly it offers the means to infer new knowledge from the existing one.

\subsection{Multimedia standardised frameworks}

MPEG-7 MDS (Multimedia Description Schemes) [6] is an open standard composed by a set of Description Tools, namely Descriptors and Description Schemes. The Media Description Tools can be used to thoroughly describe multimedia resources, including technical and low-level 
features such as audiovisual encoding format, spatial and temporal resolution, colour space. etc.

MPEG-21 DIA (Digital Item Adaptation) [7] is one of the most complete emergent standards addressing the use of context. DIA specifies tools that may be used to assist the adaptation of complex digital media objects, designated as Digital Items (DIs). The Usage Environment Description (UED) tool from DIA enables to describe the characteristics and capabilities of networks, terminals and environments as well as preferences of users. DIA also allows to describe operations that can be performed upon the content and the result that can be expected.

\subsection{Ontology and rules inference standards}

The Web Ontology Language (OWL) [8] is a formal language for defining, publishing and sharing machineinterpretable semantic content on the Web. Three OWL species of increasing expressive power are specified: OWL Lite, OWL DL (Description Logics), and OWL Full. The most common feature of ontologies is the creation of classes, for representing sets of individuals sharing the same characteristics. It also declares properties, representing the features of the class individuals, and provides domain and range constraints for these properties. OWL can state that a property is functional, transitive, symmetric, or the inverse of an existent object property. Moreover, OWL is able to provide restrictions on how properties behave, including type restrictions, cardinality restrictions, and value restrictions. Based on a combination of OWL and RuleML (Rule Markup Language), SWRL (Semantic Web Rule Language) [9] extends the set of OWL axioms to include Horn-like rules. This specification adds rules to OWL ontologies, while also providing an extra layer of expressivity and the capability to infer additional information from an OWL knowledge base. This is most fruitful to express decision rules when building domainspecific knowledge for context-aware systems.

\section{THE ADAPTATION DECISION ENGINE (ADE) ARCHITECTURE}

The ADE is the sub-system of the VISNET-II context-aware content adaptation platform, responsible for deciding how to react to changes in the usage environment, in a way that best meets users' expectations. The architecture of the ADE middleware is illustrated in Fig. 1.

This architecture is designed based on a multi-module service-oriented approach. Low-level context information, in the form of MPEG-21 DIA and MPEG-7 descriptors, is gathered by a Context Acquisition Processing sub-system which interacts with the Context Service Manager. The latter, processes and saves the aforementioned context in the Context Knowledge Base (KB) for knowledge sharing and reuse. When dealing with protected content, it also requests contextual information related to adaptation authorisation.

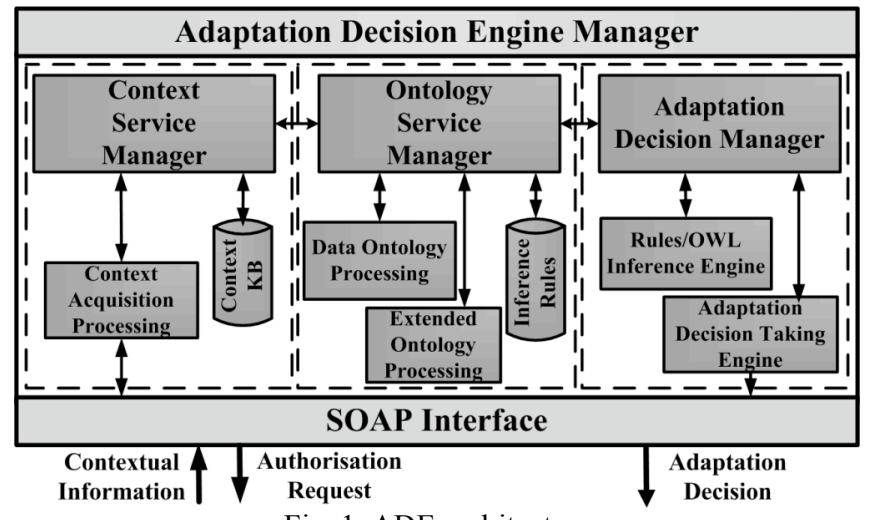

Fig. 1. ADE architecture

The Ontology Service Manager is responsible to integrate the gathered knowledge with the concepts and inference rules defined in the MULTICAO core and associated extended layer ontology. This operation is requested to the Data Ontology and Extended Ontology Processing sub-modules. Whenever new or updated contextual information is available, the Rules Inference Engine is invoked and interacts with the Decision Taking Engine to select the most appropriate adaptation and corresponding service parameters. The Adaptation Decision Engine Manager, at the application level, is responsible for the session management and modules invocation.

\section{CONTEXT-AWARE ONTOLOGY FOR MULTIMEDIA APPLICATIONS (MULTICAO)}

The Context-Aware Ontology for Multimedia applications (MULTICAO) was designed to provide the support for context-aware and DRM-enabled multimedia content adaptation decision taking operations. The ontology is divided into a core context ontology layer, describing the common knowledge denominator under different real world situations of multimedia content consumption and an extended context ontology layer which is domain-specific and only relevant for the application under consideration.

MPEG-21 DIA is the reference standardisation to describe the usage environment in a multimedia consumption scenario. Whilst providing a comprehensive structured representation of low-level context, the expressiveness of DIA tools is not semantically rich to describe complex real world situations where contextual information is interconnected. We also found that user visual preferences are still not fully considered in the UED descriptors. Likewise, it is not the most suitable format to enable automatic computer reasoning neither to be re-usable and interoperable with other domains. In order to overcome these limitations, we have developed a core ontology based on the DIA-UED tools The MULTICAO ontological model is shown in Fig. 2. It is developed using OWL DL, which provides decidability and complete reasoning mechanisms support to make automatic inferences over the knowledge base. 


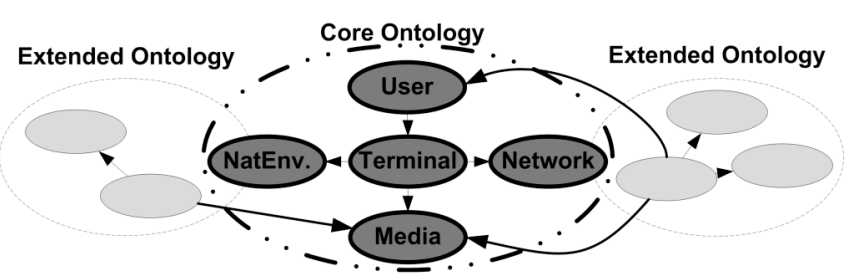

Fig. 2. MulTICAO ontological model

The ADE thus supports interoperability of OWL with the MPEG-21 DIA in a way that the usage environment descriptors can be transparently integrated in the developed ontology.

\subsection{Core Ontology}

The core context knowledge base provides descriptions of generic concepts and rules that can be used and shared in any multimedia consumption scenario. Thus, the core ontology is the basis for interoperability support between OWL and multimedia descriptors.

As depicted in Fig. 2, the core layer defines five basic concepts. The conceptualization of the profiles based on the UED tool, namely user, terminal, network and natural environment concepts, are detailed in [10]. Additionally the core ontology includes the description of a media profile derived from MPEG-7 MDS, capturing the low-level audiovisual features, which characterises the content being consumed. The domain knowledge thus obtained can be used to model domain rules and heuristics that help to improve the quality and effectiveness of adaptation decision.

\subsection{Extended Ontology Layer}

The extended context ontology layer can be seen as a set of different, domain-specific ontologies. It enables to build a context-aware system customised to specific application scenarios. Using knowledge captured by the core ontology, reflecting changes in the sensed context, it provides the system the ability to formulate an adaptation decision suitable to the specific scenario and to the sensed changes.

One specific application scenario covered by our work, is the consumption of protected content [10]. Accordingly, an ontology for representing content adaptation authorisation mechanisms, whose conceptual model can be depicted in Fig.3, was developed as part of the extended layer. This provides the means to simulate a governed network, where adaptation operations are only performed if they do not violate any condition expressed in DRM licenses. Additionally, only the permitted adaptation operations and associated change constraints are instantiated in the ontology restricting the adaptation decision possibilities.

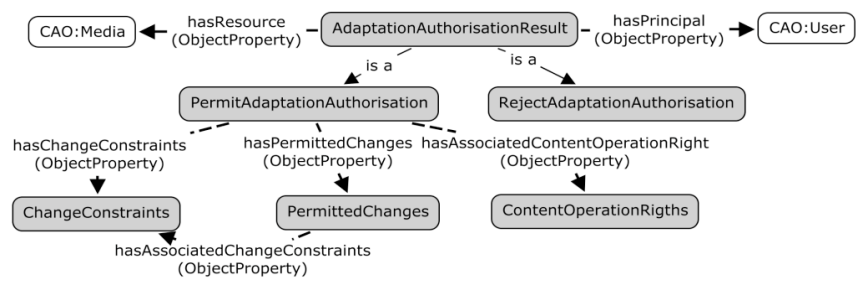

Fig. 3. Extract of the Adaptation Authorisation Extended Ontology

The extended layer also includes a second ontology, representing the semantics of the intelligent part of the context-aware system, by providing the necessary SWRL rules to conduct the adaptation decision taking process. The rule, shown in Table I, examines if the multimedia content is suitable to be reproduced on the actual network conditions, based on media bit rate and sensed network available bandwidth. If not, it will trigger a necessary content adaptation using media bit rate transcoding or discarding scalability layers, depending on the available adaptation engine.

Table I. SWRL adaptation decision rule example

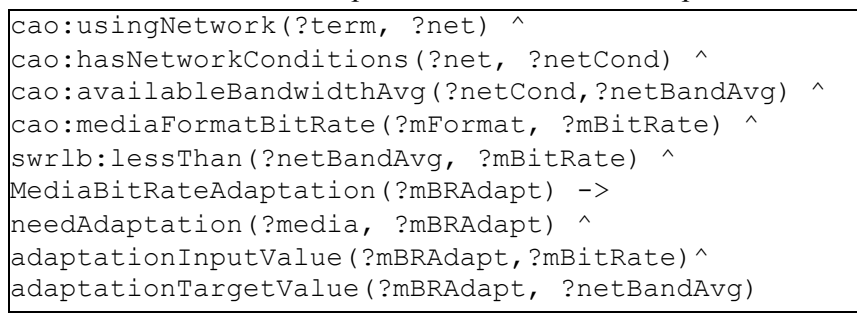

\subsection{Rules inference mechanism}

Using a set of rules in the extended ontology, which are specific to the application scenario, enables to infer realword situations that are indeed likely to occur in those specific applications. This inference process requires a rulebased engine module to infer additional information according to the rules inference and triggering.

Whenever some new facts are inferred by the rule-based engine, the results are inserted into the extended ontology. Once the outcome from the inferences is obtained, the domain application is in charge of the execution of the required actions to take an adaptation decision. Due to the complexity of the multimedia content consumption scenario, several conflicting rules can be inferred and derive an ambiguous adaptation decision. For instance, the user preferences may indicate to consume the multimedia content at its best possible quality but the terminal has not the necessary screen dimensions or the network conditions obligate to reduce the bit rate. Thus, before any attempt to consider overridden adaptation operations, the domain application must provide a priority decision algorithm to best suit the user experience and ensure that no conflicting adaptation operations are passed to the adaptation engines. 


\section{APPLICATION OF THE MULTICAO}

A field worker equipped with a PDA and standing in a noisy place is engaged in a remote Virtual Collaboration (VC) session. Not being able to access a wireless Internet link, he is connected over a cellular mobile network. Moreover, the remaining device battery lifetime does not seem to be enough for viewing the full session. An extract of the usage environment contextual information received by the $\mathrm{ADE}$ is shown in Table II whilst the information related to the video stream format from the VC session is shown in Table III. This information is instantiated into the core ontology and the adaptation decision taking is now possible using the extended ontology.

Table II. Usage environment contextual information

\begin{tabular}{|l|l|l|}
\hline \multirow{3}{*}{ Terminal } & Display Size & QCIF $(176 \times 144)$ \\
\cline { 2 - 3 } & Maximum Frame Rate & $25 \mathrm{fps}$ \\
\cline { 2 - 3 } & Battery Time Remaining & 15 minutes \\
\hline Network & Available Bandwidth & $128 \mathrm{kbps}$ \\
\hline Environment & Noise Level & $80 \mathrm{~dB}$ \\
\hline \multirow{2}{*}{ User } & Frame Rate Preference & $30 \mathrm{fps}$ \\
\cline { 2 - 3 } & Frame Size Preference & VGA $(640 \times 480)$ \\
\hline
\end{tabular}

Table III. Media stream format information

\begin{tabular}{|l|l|l|}
\hline Visual Coding & Frame Rate & $30 \mathrm{fps}$ \\
\cline { 2 - 3 } & Frame Size & VGA $(640 \times 480)$ \\
\hline & Media Bit Rate & $1024 \mathrm{kbps}$ \\
\hline
\end{tabular}

Tables II and III clearly show that some form of content adaptation is needed to provide a service with acceptable quality, while satisfying the restrictions. Using the knowledge built by the core ontology from the data presented in tables II and III the ADE is able to select a set of possible adaptation operations, namely spatial and temporal scaling, sound level increase, etc.

Additionally, the extended ontology layer provides information specific to the VC session. Such information can include the indication that the worker will benefit more from receiving a good spatial resolution video without caring too much about the temporal resolution and that video and written indications would be preferable to audio. In other application areas, as for example videosurveillance, it could be preferable to have the full motion video, sacrificing the image quality. Responding to these constraints and specific rules, the ADE decides to decrease the temporal resolution of the video, to decrease the spatial resolution but only such as to satisfy the terminal constraints, to summarise the video focussing only on the parts that are essential to conduct his work and finally to eliminate the audio.

Table IV. Adaptation decision parameters

\begin{tabular}{|l|l|l|}
\hline & Input Values & Target Values \\
\hline Spatial resolution & $640 \times 480$ & $176 \times 144$ \\
\hline Frame Rate & $30 \mathrm{fps}$ & $15 \mathrm{fps}$ \\
\hline Bit Rate & $1 \mathrm{Mbps}$ & $128 \mathrm{Kbps}$ \\
\hline Volume Level & 0.85 & N/A \\
\hline
\end{tabular}

\section{CONCLUSIONS}

Adaptation can be seen as an effective solution to allow the consumption of multimedia content under usage conditions that prevented its consumption in its original form. Whereas several solutions have already been proposed to assist the decision to select the most appropriate type of adaptation, those approaches usually take into consideration low-level information individually, without looking at relations between that data, nor at dependencies with the actual application and real-world situation the user is engaged in. The same low-level context may provide different indications depending on the application. The use of a twolevel ontology and specific inference rules, as proposed in this paper, can greatly enhance the adaptation decision engine, providing the means to overcome these limitations and thus take decisions that better meet users' expectations.

\section{ACKOWLEDGEMENTS}

The work here presented has been developed within VISNET II, a European Network of Excellence, partially funded under FP6-IST. The authors would like to thank their colleagues in VISNET for their productive collaborations.

\section{REFERENCES}

[1] D. Jannach, K. Leopold, C. Timmerer, and H. Hellwagner. "A knowledge-based framework for multimedia adaptation," Applied Intelligence, Springer Verlag, vol. 24, no. 2, pp. 109125, Apr. 2006.

[2] F. Lopez, and J.M. Martinez, "Multimedia Content Adaptation Modelled as a Constraints Matching Problem with Optimisation," in Proc. WIAMIS2007, June 2007.

[3] D. Rios, P. Dockhorn, G. Guizzardi, L. Ferreira, J. Pereira, and M. van Sinderen, "Using ontologies for modeling contextaware services platforms," Workshop on Ontologies to Complement Software Architectures, Oct. 2004.

[4] T. Gu, H. K. Pung, and D. Q. Zhang, "A service-oriented middleware for building context-aware services," J. Netw. Comput. Appl., vol. 28, no. 1, pp. 1-18, Jan. 2005.

[5] W. Qin, Y. Shi and Y. Suo, "Ontology-based context-aware middleware for smart spaces," Tsinghua Science \& Technology, vol. 12, no. 6, pp. 707-713, Dec. 2007.

[6] "Information Technology - Multimedia content description interface - Part 5: Multimedia description schemes," ISO/IEC Standard ISO/IEC 15938-5:2003, 2003.

[7] "Information Technology - Multimedia Framework (MPEG21) - Part 7: Digital Item Adaptation," ISO/IEC Standard ISO/IEC 21000-7:2007, Dec. 2007.

[8] Web Ontology Language (OWL): Overview, W3C Recommendation, Feb. 2004. [Online]. Available: http://www.w3.org/TR/owl-features/.

[9] SWRL: A Semantic Web Rule Language Combining OWL and RuleML, W3C Submission, May 2004. [Online] Available: http://www.w3.org/Submission/SWRL/.

[10] V. Barbosa, A. Carreras, H. K. Arachchi, S. Dogan, M. T. Andrade, J. Delgado, and A. M. Kondoz. A scalable platform for context-aware and DRM-Enabled adaptation of multimedia content. In ICT-MobileSummit, June 2008. 